of surface conditions in the distortion of an elastic solid, with the treatment of capillarity, of vortex-motion, and of discontinuous fluid motion (Flüssigkeitsstrahlen).

Besides these definite classes of papers, there is a number of noteworthy memoirs of a more miscellaneous character :-on important propositions in the Thermodynamics of solution and vaporization, on crystalline reflection and refraction, on the influence of heat conduction in a special case of propagation of sound, on the optical constants of Aragonite, and on the Thermal Conductivity of Iron.

Finally we have the series of papers on Radiation, partly mathematical partly experimental, which, in 1859 and 1860 , produced such a profound impression in the world of science, and which culminated in the great work on the solar spectrum whose title is given above. The history of Spectrum Analysis has, from that date, been one of unbroken progress. Light from the most distant of visible bodies has been ascertained to convey a species of telegraphic message which, when we have learned to interpret it, gives us information alike of a chemical and of a purely physical character. We can analyze the atmosphere of a star, comet, or nebula, and tell (approximately at least) the temperature and pressure of the glowing gas. But, at the present time, the fact that such information is attainable is matter of common knowledge.

This is not an occasion on which we can speak of questions of priority, even though we might be specially attracted to them by finding v. Helmholtz and Sir W. Thomson publicly taking (in full knowledge of all the facts) almost absolutely antagonistic views. However these points may ultimately be settled, it is certain that Kirchhoff was (in 1859) entirely unaware of what Stokes and Balfour Stewart had previously done, and that he, with the powerful assistance of Bunsen, MADE what is now called Spectrum Analysis : Kirchhoff, by his elaborate comparison of the solar spectrum with the spectra of various elements, and by his artificial production of a new line whose relative darkness or brightness he could vary at pleasure; Bunsen by his success in discovering by the aid of the prism two new metallic elements.

P. G. TAIT.

\section{ON THE SIGNIFICATION OF THE POLAR} GLOBULES.1

T has long been known that the egg of some animals, after becoming mature and before undergoing its embryonic development, throws out certain bodies of globular form, which take no part in the embryonic development, but perish sooner or later. These polar globules have been found on the eggs of nearly all classes of animals, and it has been proved that they are real cells, composed of nucleus and cell-body.

Several theoretical opinions have been expressed in regard to their signification. Some naturalists believe them to be only a kind of excretion of the egg; others even think them to be of no functional importance, and perceive in them only a remnant of some ancestral process, a recapitulation of some ancient part of the phylogenetic development.

Now it is true that, in many animals, structures occur without any physiological value, but it is also known that such structures-as, for instance, the hind-legs of whalesdisappear more and more in the lapse of phylogenetic development. Furthermore, such rudimentary organs never disappear in all species and genera of a large group simultaneously, but in one genus or species they persist longer than in another. Thus, some whales possess certain of the bones of their hind-legs lying between the muscles of the trunk, whilst others have preserved only one bone of

x Paper read by Prof. August Weismann before the British Association at Manchester. the pelvis. Now the polar globules might have been regarded as insignificant and rudimentary as long as they were only known in a few groups of the animal kingdom. But as their existence is now proved in nearly all classes of animals, and as they appear in all of them in the same manner, we are compelled to assume that they possess at least some physiological significance.

Mr. Sedgwick Minot and your illustrious Balfour made a great step forward in attempting--each independently of the other-to attribute a high importance to the expulsion of the polar globules. As you know, they suggested that the egg-cell was originally herimaphrodite, and that the polar globules were the male portion, which had to be thrown off. They based their idea upon the generally accepted view, according to which fecundation is the union of a specific male with a specific female substance. If this is true, then the fecundated ovum contains both these substances in equal quantities; and the observations upon the segmentation of the egg lead further to the conclusion of $\mathrm{E}$. Van Beneden, that all cells of the body contain these two substances, and that they are all hermaphrodite. The throwing out of polar globules was, according to these views, the means of preventing parthenogenesis, which must have occurred if the male substance had remained in the egg. This was Balfour's opinion, and he formulated the same with all precaution, putting it forward as a supposition, which might prove true or not. He himself even pointed out the way by which a decision could be obtained, in his statement, that, if his theory was true, polar globules would not be found in parthenogenetic eggs. Certainly, if polar globules represent the male substance, they cannot be thrown out in an egr that is not destined to be fertilized, and which therefore would not receive the male substance from another celi

Now, I have tried to decide this question by observing whether parthenogenetic eggs throw out polar globules or not, and I discovered several years ago that polar globules certainly exist in parthenogenetic eggs. I have found them in the summer egos of Daphnidæ, and later, assisted by my pupil Mr. Ishikava, of Tokio, I have also found them in the parthenogenetic eggs of Cypridæ and of Rotatoria.

Now it is impossible that these polar globules can contain the male part of the egg. and the question arises, What other significance can be attributed to them?

When I ascertained the facts which I have just described, I was not at the time aware of another fact that I am about to lay before you, and which seems to me to possess an important bearing upon the meaning of polar globules, and of sexual propagation in general. This fact is a very simple one: Parthenogenetic eggs throw out only one polar body, whilst sexual eggs throw oui two of them.

The importance of this fact lies in the significance of the substance that is thrown out in the polar globules or polar cells. You know well that it is a true cell-division which leads to the formation of polarglobules, and that the first polar cell takes away from the egg-cell one-half of the nuclear substance. You are also aware that the second polar cell again takes away half of the nuclear substance remaining in the egg. Hence in sexual eggs three-quarters of the nuclear substance originally contained in the egg-cell are taken away by the two polar cells. In parthenogenetic eggs only one polar cell is formed, and consequently only one-half of the original mass of nuclear substance is removed from the egg-cell.

Now you know that nuclear ubstance is a very im portant thing. The experiences and reflexions of the last ten years have led to the general conviction that nuclear substance is the part that controls the whole cell, and that the entire structure as well as the functions of the cell depend upon its minute structure. The nuclear substance is the idioplasma of the botanist Nägeli. Upon the molecular structure of it the form and function of every 
cell in the body depend, and consequently the form and function of the whole body are determined by the nuclear matter or idioplasma of the first cell, the egg-cellparthenogenetic or fertilized.

If this theoretical view is correct, then we must be astonished that so much of this important nuclear substance is lost to the egg-cell-namely, one-half by the parthenogenetic ovum, and half as much again by the sexual one. What can be the cause that renders it necessary for this to happen before the egg-cell is able to develop into an embryo?

I will give a short account of my ideas upon the subject.

(I) The nuclear substance or idioplasma of the first polar body must be detrimental to the further development of the egg, for it is thrown out in all kinds of eggs, parthenogenetic as well as sexual, and the embryonic development never begins before the first polar cell has been expelled. Now, if the nuclear substance truly controls the cell and compels it to take a certain shape and a certain histological structure, there must be such a substance, such an idioplasma, also in the youngest eggcells. This idioplasma causes the egg to develop a yolk possessing a certain colour and structure, it causes the egg to form a shell of a certain thickness and structure; briefly, it compels the young egg-cell to attain a degree of histological differentiation which it did not previously possess. For the youngest egg-cells are essentially similar in most animals, whilst mature egg-cells are very different and can often be very well distinguished in different species. The specific idioplasma of the grow ing egg-cell-I call it ovogenetic idioplasma-cannot be the same as that contained in the nucleus of the mature egg, and which controls the development of the embryo. It cannot be that idioplasm 1 which determines the development of a certain e rg cell into a duck and not into a swan; it cannot be that kind of idioplasma which I have called germ-idioplasma, or simply germ-plasma.

Of course there must also be germ-plasma in the young egg-cell; I believe that in the youngest germ-cells there is no other idioplasma than germ-plasma, and that this germplasma changes into ovogenetic plasma, only a very small part of germ-plasma remaining unaltered.

This remaining part grows with the growth of the egg, and finally attains the same volume as the ovogenetic idioplasma. Then the division of the nuclear substance takes place, and the superfluous ovogenetic substance is removed in the first polar globule, whereupon the egg-cell contains only germ-plasma.

This is my explanation of the removal of the first polar cell.

(2) In regard to the second it is clear that an egg that contains only germ-plasma should be capable of undergoing embryonic development, unless the quantity of germ-plasma should prove to be too small. But this is not the case. Parthenogenetic eggs enter upon embryonic development immediately after the expulsion of the first polar globule. Sexual eggs do not thus develop, and we have to inquire into the reason for this. I believe it is because they throw out a second polar cell, which takes away one-half of the germ-plasma left within the egg-cell. After this the quantity of germ-plasma is too small for entering upon embryonic development, and therefore the egg-cell remains undeveloped, unless the lost quantity of germ-plasma be replaced in the process of fertilization. Embryonic development takes place immediately after the union of the germ-plasma of a spermatozoon with the remaining germ-plasma of the ovum. Consequent upon this the quantity of germ-plasma in a fertilized egg again becomes equal to that which was present after the separation of the first polar globule, and also equal to that which enters upon embryonic development in the parthenogenetic egg.

This is perfectly simple, but a great difficulty remains.
Why is it necessary that the sexual egg should throw out half of its germ-plasma; why does it not retain the whole quantity of this important substance?

You would perhaps answer, Because the quantities of male and of female germ-plasma, that are united by fecundation, must be equal. Indeed, the facts of heredity lead to the opinion that these two kinds of germ-plasma must be equal in quantity, and we have microscopical observations recorded by Van Beneden, Carnoy, and others, which further support this conclusion. But if the quantity of germ-plasma must be equal in both, why should the germ-plasma of the egg increase so largely as to attain twice the volume of the germ-plasma of a spermatic cell? Nature is not so wasteful as to throw away so important a substance for nothing. There must be an adequate cause why in sexual eggs the germ-plasma must be halved before fecundation can take place.

I believe I can point out the reason why this is necessary, but before I do so I must beg you to first enter with me upon a few theoretical considerations on the subject of heredity.

Heredity depends upon the germ-plasma, as I have said before; the minute molecular structure of the germplasma causes the egg-cell to develop into a duck or a swan, it also causes the egg to develop into a Negro or into a European, into a Mr. Smith or into a Mr. Jones; in short, all qualities of the developed individual depend upon the constitution of this germ-plasma. In my opinion sexual propagation implies the union of two different germ-plasmas to form the single nucleus of the egg-cell; and the two substances that are united in the process of fertilization I believe to be equal in size and quantity.

Now let us suppose that we lived at a time when sexual propagation had not yet existed, and that we were present at its origin. We should then observe the union of two different germ-plasmas, both of the same size and quantity, but of a slightly different molecular constitution, one coming from one parent and the other coming from another. Both substances must be thoroughly homogeneous-that is to say, they must be composed of particles that are equal in their chemical, molecular, and morphological constitution. Let us illustrate this by a diagram, in which we represent each germ-plasma as a thread or a loop, which we know to be the microscopical form of germ-plasma and of nuclear plasma in general. For simplicity's sake we will represent only one loop for the germ-plasma of each parent. We have then two loops, the first representing the peculiarities of the germplasma of one parent, and the second representing the peculiarities of the other parent, and we will discriminate between them by making the first green and the second red.

These two individual kinds of germ-plasma unite and form together the nucleus of the fertilized egg, which develops into a new individual of the second generation. This individual will form again germ-cells, and each of these germ-cells will contain a germ-plasma, which is not homogeneous, as before, but composed of two halves, derived respectively from the two parents. In each succeeding generation the germ-plasma must attain to a more complicated constitution, it must contain twice as many different kinds of germ-plasma as were contained in the germ-plasma of the preceding generation. If we follow this development of the germ-plasma for a few generations, we shall find that union will take place by sexual propagation between the germ-plasmas of two individuals of the second generation, each containing two different kinds of germ-plasma. In this way the individuals of the third generation will be formed possessing germ-cells which contain four different kinds of germplasma. I have called these different kinds of germplasma Ahnenplasma, a word that can be rendered in English by the term ancestral plasma. By sexual propagation the individuals of the third generation would give 
rise to individuals of the fourth generation, and the germcells of these last individuals must contain eight different ancestral plasmas; similarly the germ-cells of the fifth generation must contain sixteen ancestral plasmas, and so on. It is thus clear that in a very small number of generations the composition of the germ-plasma must become extremely complicated: by the tenth generation it would already contain 1024 different ancestral plasmas.

We do not know how far this may go, because we do not know how small are the primary elements of germplasma, nor do we know how many of these elements may be indispensable for the youngest and smallest germ cells. But if we imagine these elements to be excessively small, this process of doubling the number of ancestral plasmas in each generation must have come to an end after a certain number of generations, whether they were IO, 20 , 100 , or 1000 !

From the time at which the germ-plasma first attainer its utmost complexity further sexual propagation was only possible by halving the number of ancestral plasms contained in the germ-plasma. Clearly, this process of halving ought to take place in male germ-cells as well as in female ones, but at this moment we are only sure of its existence in the latter. We have seen that one-half of the germ-plasma contained in the nucleus of the egg-cell is expelled in the second polar cell. That the nuclear substance thus expelled is true germ-plasma, is not a mere supposition, but a certainty. We know of developing eggs which are either fertilized or unfertilized, and in the latter case they develop by parthenogenesis. Such are the eggs of the honey-bee. We may assume that if these eggs remain unfertilized they will expel only one polar globule, but that if, on the other hand, they are penetrated by a spermatozoon they will also expel the second globule. Thus the same idioplasma that is expelled from the fertilized egg remains, and forms half of the first segmentation-nucleus in the parthenogenetic egg. It must therefore be true germ-plasma.

I do not doubt that this is the true significance of the formation of a second polar globule. We can see the necessity on theoretical grounds for the removal of half the number of ancestral germ-plasmas; and we actually find that half of the germ-plasma is removed in every sexual egg.

If this reasoning be correct, our views on sexual propagation must undergo a total change. Fertilization is no longer an unknown impulse given to the egg-cell by the entrance of a spermatozoon, but it is simply the union of the germ-plasmas of two individuals. The spermatozoon is no longer the spark which kindles the powder, or the relatively small force which converts potential into actual energy, but it is merely the carrier of germ-plasma of a certain individual, possessing the necessary qualities for reaching, penetrating, and fusing with the bearer of germplasma from another individual. There are no essential, but merely individual, differences between the nuclear substance of the spermatozoon and that of the ovum. There are no such things as male or female nuclear substances, but merely male and female cells, carriers of the immortal germ-plasma. The differences are wholly individual and of merely secondary importance, and nothing corresponding to the ordinary notions implied by the terms male and female exists in germ-plasma.

If this be so, then it is clear that the fact of sexual propagation demands a new explanation. We must attempt to explain the reason why Nature has in sisted upon the rise and progress of sexual propagation. If we bear in mind that in sexual propagation twice as many individuals are required in order to produce any number of descendants, and if we further remember the important morphological differentiations which must take place in order to render sexual propagation possible, we are led to the conviction that sexual propagation must confer immense benefits upon organic life. I believe that such beneficial results will be found in the fact that sexual propagation may be regarded as a source of individual variability, furnishing material for the operation of natural selection. I believe that sexual propagation has become prevalent among the higher organisms for the purpose of conserving and multiplying that individual variability which owes its first origin to the Protozoon condition of such higher organisms. But it is not now my purpose to speak further upon this subject : I have already discussed it elsewhere ("Die Bedeutung der sexuellen Fortpflanzung für die Selections-Theorie," Jena, I886).

Whatever is to be said for the above hypotheses, the facts I have the honour of bringing before you to-day seem at least to prove that sexual propagation depends on the removal of half of the germ-plasma of the egg and the replacement of it by the same quantity of germ-plasma of another individual. This is now a fact which may be regarded as indisputable; and, further, the existence of true parthenogenesis is now proved beyond doubt. For we know now that an egg which expels only one polar globule enters without delay into embryonic development, inasmuch as it has retained the whole of its germplasma.

\section{THE TOTAL ECLIPSE OF LAST AUGUST IN} IAPAN.

THE eclipse has come and gone, and our little party is on its way home with a few papers and a small box of glass plates-a rather meagre showing for the hard work of our summer months. Although we were so unfortunate as to have uninterrupted cloud throughout the entire duration of the eclipse, our expedition to Japan has not been so dismal a failure all told. Apart from sundry observations of minor importance contributed by volunteer observers at scattering stations for whom I had prepared instructions, Dr. W. J. Holland, who joined the Expedition at my invitation as naturalist, has been actively engaged in botanical and entomological research in fruitful fields, and has a good harvest to report. $\mathrm{He}$ has also valuable notes upon his ascent of Nantaisan, Asamayama, and Nasutake (which latter he appears to have been the first foreigner to ascend); while the separate expedition to the summit of Fuji-san ( 12,400 feet), which I had the pleasure to carry out under the auspices of the Boyden Fund of the Harvard College Observatory, and on which I had the highly-valued co-operation of Dr. E. Knipping, Meteorologist of the Japanese Weather Service, resulted, among other things, in the determination of its rare fitness as a site for astronomical observation-of which more elsewhere.

With reference to the preliminaries of the Eclipse Expedition it is necessary to state that early in the present year the trustees of the Baclie Fund of the National Academy of Sciences, Washington, made a grant to Prof. Newcomb, the Superintendent of the Nautical Almanac, for observing the total solar eclipse of August 19, I887. Prof. Newcomb determined the general lines of research to be undertaken, decided upon locating the observingstation in Japan, and placed me in charge of the Expedition. After some weeks of preparation in Washington and elsewhere, I set out for Japan on June 9 , and arrived in Yokohama a month later. There was no small difficulty attending the definitive location of the instruments for observing the eclipse, owing to the deficiency of precise meteorological information regarding the region of the shadow-path. All existing data were kindly placed at my disposal by the officers of the Japanese Government, and several of the departments contributed in other ways to the assistance of an expedition which, had the skies been favourable, could not have failed of entire success.

Going northward from Tokio along the line of the Japanese Railway Company, to the courtesy of whose 